\title{
A!
}

This is an electronic reprint of the original article.

This reprint may differ from the original in pagination and typographic detail.

Heinonen, J.; Bukharev, I.; Ala-Nissilä, T.; Kosterlitz, J.M.

\section{A simple model for anisotropic step growth}

Published in:

Physical Review E

DOI:

10.1103/PhysRevE.57.6851

Published: 01/01/1998

Document Version

Publisher's PDF, also known as Version of record

Please cite the original version:

Heinonen, J., Bukharev, I., Ala-Nissilä, T., \& Kosterlitz, J. M. (1998). A simple model for anisotropic step growth.

Physical Review E, 57(6), 6851-6858. https://doi.org/10.1103/PhysRevE.57.6851

This material is protected by copyright and other intellectual property rights, and duplication or sale of all or part of any of the repository collections is not permitted, except that material may be duplicated by you for your research use or educational purposes in electronic or print form. You must obtain permission for any other use. Electronic or print copies may not be offered, whether for sale or otherwise to anyone who is not an authorised user. 


\title{
Simple model for anisotropic step growth
}

\author{
J. Heinonen, ${ }^{1}$ I. Bukharev, ${ }^{2}$ T. Ala-Nissila, ${ }^{1,2,3},{ }^{*}$ and J. M. Kosterlitz ${ }^{2}$ \\ ${ }^{1}$ Helsinki Institute of Physics, P.O. Box 9, FIN-00014 University of Helsinki, Helsinki, Finland \\ ${ }^{2}$ Department of Physics, Brown University, Box 1843, Providence, Rhode Island 02912-1843 \\ ${ }^{3}$ Laboratory of Physics, Helsinki University of Technology, P.O. Box 1100, FIN-02150 HUT, Espoo, Finland
}

(Received 7 November 1997; revised manuscript received 11 February 1998)

\begin{abstract}
We consider a simple model for the growth of isolated steps on a vicinal crystal surface. It incorporates diffusion and drift of adatoms on the terrace, and strong step and kink edge barriers. Using a combination of analytic methods and Monte Carlo simulations, we study the morphology of growing steps in detail. In particular, under typical molecular beam epitaxy conditions the step morphology is linearly unstable in the model and develops fingers separated by deep cracks. The vertical roughness of the step grows linearly in time, while horizontally the fingers coarsen proportional to $t^{0.33}$. We develop scaling arguments to study the saturation of the ledge morphology for a finite width and length of the terrace. [S1063-651X(98)04506-1]
\end{abstract}

PACS number(s): 81.15.Hi, 68.35.Fx, 82.20.Wt

\section{INTRODUCTION}

Atomistically controlled growth of metal and semiconductor crystal surfaces constitutes an important field of research both from technological [1] and fundamental theoretical [2] points of view. Among all the different growth techniques, molecular beam epitaxy (MBE) has a special status since it can be very efficiently used to produce growth in a well defined layer-by-layer growth mode. Experiments using the reflection high-energy electron diffraction technique [3] indicate two main mechanisms of growth in such cases: layer growth by nucleation and spreading of twodimensional (2D) islands on a nominally flat substrate, and step-flow growth of a substrate with steps. In the latter case, it is crucial to be in the regime where the flux of adatoms is small enough, and their diffusion fast enough to avoid island nucleation on terraces. Such a window of the relevant physical parameters may be found experimentally for many materials [1].

An important practical realization of the step-flow situation is epitaxial growth on a vicinal surface that is cut in a direction slightly off from a high-symmetry one. Such surfaces often consist of broad terraces of size separated by monatomic steps at distance $l$ from each other. By changing the miscut angle, the density of the steps and thus $l$ may be controlled. The physics of MBE growth on such surfaces can be in the simplest terms described by the following schematic model (see Fig. 1). There is a flux $F$ of adatoms that impinges upon the terraces. Particles on terraces then diffuse around with an associated diffusion constant $D$, and may be desorbed after a time $\tau$. Upon approaching step edges, particles can either cross them from above or below, be reflected from them, or be incorporated into the growing ledge. Attachment is usually characterized by Arrhenius-type rate constants $k_{+}$and $k_{-}$, which refer to the average rates of

\footnotetext{
*Author to whom correspondence should be addressed. Electronic address: alanissi@csc.fi
}

particles arriving at the ledge from below or above, respectively.

This simplified picture of step-flow growth was first introduced by Burton et al. [4]. More recently, attention has been drawn to the fact that in many real systems, $k_{+}$and $k_{-}$need not be equal [5] because of the existence of step edge barriers [6]. These barriers may often be present at step edges due to reduced coordination of atoms. Recent theoretical work shows that the step barriers play an important role in controlling growth under MBE situations $[7,8]$. In particular, if these barriers are high, adatoms cannot cross steps, and the particle current will be in the direction of ascending steps. For vicinal surfaces, this stabilizes the step-flow growth mode when nucleation on terraces is neglected. If the average distance between nucleation centers is $l_{N}$, step-flow growth requires that $l / l_{N} \ll 1$.

Most of the recent work dealing with step growth has concentrated on the global properties and kinetic roughening of growing surfaces with steps under MBE conditions $[1,2,9]$. However, attention has also been paid to the properties of individual steps under growth [10-14]. It is a well known property of ideal, isolated 1D steps that they are thermally rough above zero temperature due to kinks. Using linear stability analysis, Bales and Zangwill [11] have shown

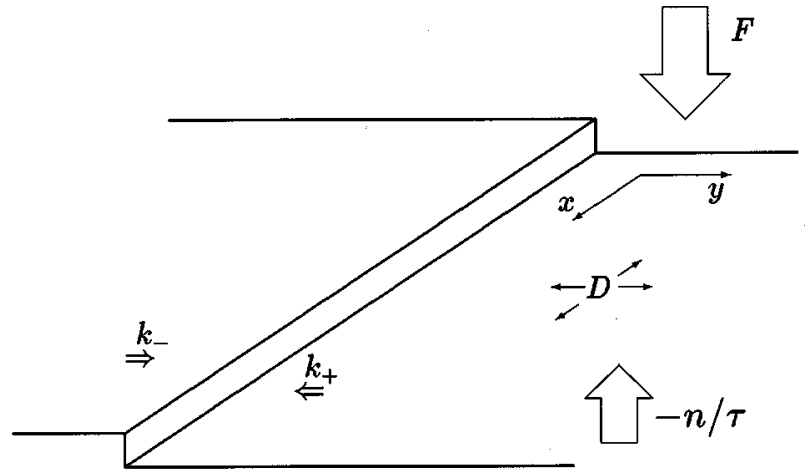

FIG. 1. A schematic view on adatom dynamics on a vicinal surface. The local adatom concentration is denoted by $n$ and the other symbols are explained in the text. 
that in a system with unequal attachment rates $k_{+} \neq k_{-}$, a straight terrace ledge can become unstable when $k_{+}>k_{-}$. This kind of growth-driven instability is particularly interesting since it may lead to the appearance of "wavy" patterns of the ledges. More recently, Salditt and Spohn [12] have argued that in addition to the instability, there is a regime for isolated steps (in the case of strong step-edge barriers) where the Kardar-Parisi-Zhang (KPZ) [15] theory of kinetic roughening is valid. In this regime, the width of the ledge eventually roughens as $t^{1 / 3}$ in analogy to many $1 \mathrm{D}$ surface deposition models [2].

In this work, we study the nature of ledge or step-edge morphologies, and the question of their roughening behavior in a simple but nontrivial model of isolated steps. This model is in part motivated by the energetics of adatoms on $\mathrm{Si}(001)$ surfaces with widely separated steps. In the model, we assume infinitely strong step-edge barriers and biased diffusion both on the terrace and at the ledge. As expected [11], the ledge always becomes morphologically linearly unstable due to the dominance of the one-sided diffusion field. Through a combination of analytic arguments and computer simulations, we show that the ledge develops fingerlike structures and its roughness grows linearly in time, in contrast to the KPZ type of roughening predicted by Salditt and Spohn [12] in the stable regime. In addition, we study the lateral coarsening of these fingers and show that it follows a $t^{0.33}$ behavior. We develop scaling arguments to study the influence of the finite width and finite length of the terrace on the growth. Finally, we discuss the relevance of these results with respect to experiments on ledge roughening under MBE growth [16].

\section{ANISOTROPIC STEP GROWTH MODEL}

\section{A. Definition of the model}

The model is defined on a two-dimensional square lattice where there is a single growing step. The average direction of the ledge is along the $x$ axis, where the width is $L_{x}$ with periodic boundary conditions. Initially at time $t=0$ the step at $y=0$ is completely straight with no thermal fluctuations present. Growth of the ledge is initiated by depositing a single particle randomly on an empty, randomly chosen terrace site in front of the ledge at $y>0$. After this, the particle performs random walk and drifts towards the ledge by jumping $l_{d}$ lattice sites in the $-y$ direction at every random walk step on the average. This means that during each step, the particle moves in the $-y$ direction with a probability $(1 / 4$ $\left.+l_{d}\right) /\left(1+l_{d}\right)$, while for the other three directions the probability is $(1 / 4) /\left(1+l_{d}\right)$.

The ledge acts as an absorbing boundary to the particle with the following rules (see Fig. 2): (i) if the particle arrives at the "top" (a section along the $x$ direction of the step), it is incorporated into it and becomes immobile; (ii) if the particle arrives at the "side" (a section along the $y$ direction of the step), it will instantaneously slide down along the ledge to the $-y$ direction until it reaches the corner site, where it is permanently incorporated into the step. These rules guarantee that the set of step heights $\{h(x, t)\}$ as measured from $y$ $=0$ obeys the solid-on-solid restriction, and the step forms a compact structure.

After the particle has been incorporated into the step, a new particle is deposited and the process is repeated. Time in

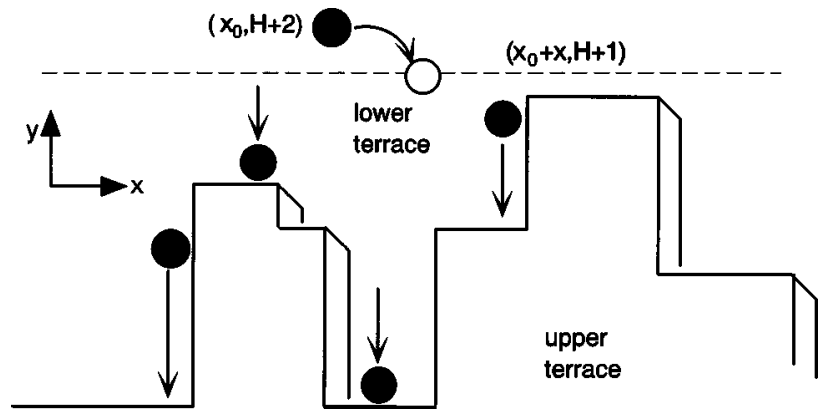

FIG. 2. Adatom dynamics in the growth model. For $L_{y}<\infty$, deposition occurs uniformly randomly at all available (unoccupied) sites, while for $L_{y}=\infty$, the particles are released from the line at $y=H+1$. Particles then diffuse on the lower terrace, and drift in the $-y$ direction. They become incorporated into the step when they either land on a top section of the step (along the $x$ axis) or slide down along the $-y$ direction to the nearest kink site, as shown schematically in the figure. If a particle attempts to cross the line at $\left(x_{0}, H+1\right)$ in the $y$ direction, it is immediately returned from $\left(x_{0}, H+2\right)$ to the line with new coordinates $\left(x_{0}+x, H+1\right)$ chosen from the spatial distribution $P_{L y}(x)$. The boundary above $y=H$ $+L_{y}+1$ (not shown) is completely reflecting.

the model is measured in terms of the average height of the growing step edge. We note that the size of the terrace in the $y$ direction is not fixed, but is chosen in such a way that the distance from the highest point of the step $H(t)$ $\equiv \max \{h(x, t)\}$ is kept at a constant value. The corresponding boundary above $y=H+L_{y}+1$ is completely reflecting and remains straight. This means that a particle at $y=H+L_{y}$ that takes a step in the $y$ direction is immediately reflected back.

An important feature of the growth model is the deposition of adatoms on the lower terrace only. This is tantamount to assuming that the step barriers are infinitely high with $k_{-}=0$ so that adatoms are reflected from a downward step leading to an average particle current in the $-y$ direction towards the up steps. The drift term $l_{d}$ is defined only in an average macroscopic sense and will depend on the deposition flux and the concentration of adatoms on the terrace in front of the step. Also, since we assume that there is no desorption of adatoms $(\tau=\infty), l_{d}$ also depends on the velocity of the step, which in turn depends on the terrace length $L_{y}$. Thus $l_{d}$ is, in principle, determined self-consistently by the other parameters of the model, but we regard it as an independent parameter that may be varied externally [17].

Finally, we would like to mention that the growth rules of the model are in part motivated by adatom dynamics on $\mathrm{Si}(001)$ surfaces with widely separated steps [18-21] under typical MBE conditions. Namely, on $\mathrm{Si}(001)$ diffusion is spatially anisotropic both on the terrace $[22,18-20]$ and at the step edges $[18,19]$. However, at least for the case of single-height steps on $\mathrm{Si}(001)$, microscopic calculations $[18,19]$ and experiments $[16,20]$ indicate that there is no significant step-edge barrier. Thus, we make no attempt to realistically model the complicated adatom dynamics in this system, since the main motivation here is to study the generic features of the unstable regime for isolated steps.

\section{B. Simulation algorithm for the model}

A straightforward Monte Carlo simulation of the growth model proposed here is in principle possible, but very diffi- 
cult for large values of $L_{y}$ and small drifts. This is because particles landing on the terrace may wander arbitrarily far from the step edge, and thus the time for a particle to become incorporated into a growing step may become very large. This problem can be solved by considering the properties of $2 \mathrm{D}$ random walkers on a finite or semi-infinite plane. For such cases, it is possible to calculate analytically the spatial and temporal probability distributions for the walkers. The idea then is that for particles that initially land on the terrace with $y>H+1$ (which is always the case if $L_{y}=\infty$ ), the simulation can be started by releasing them from an imaginary line that runs along the $x$ direction just one lattice site above the highest step, i.e., at $y=H+1$ (see Fig. 2). If the particle crosses the line again in the $+y$ direction while performing random walk, it is immediately returned to it with a new $x$ coordinate chosen from the appropriate spatial distribution, which will be derived below. In the Appendix we also calculate the mean arrival (first passage) time of a walker and indeed show that this time becomes very large for small values of $l_{d}$ and large $L_{y}$.

More specifically, to implement the simulation algorithm described above, we need to calculate the spatial probability distribution function $P_{L x, L y}(x)$, which is used to obtain the new position for a walker that crosses the line $y=H+1$ at any point. In other words, a walker crossing the line being at $\left(x_{0}, H+2\right)$ with any $x_{0}$ is put back to the new site $(x$ $\left.-x_{0}, H+1\right)$ with the probability $P_{L y}(x)$, where we assume for simplicity that $L_{x}=\infty$ (see Fig. 2). For a discrete walker, this function satisfies the recursion relation

$$
\begin{aligned}
P_{L y}(x)= & \frac{1}{2 a}\left(b \delta_{x, 0}+P_{L y}(x-1)+P_{L y}(x+1)\right. \\
& \left.+\sum_{y=-\infty}^{\infty} P_{L y}(x-y) P_{L y-1}(y)\right),
\end{aligned}
$$

where $a=2+2 l_{d}$ and $b=1+4 l_{d}$. Using the standard Fourier transformation

$$
\widetilde{P}_{L y}(k)=\sum_{x=-\infty}^{\infty} e^{i k x} P_{L y}(x),
$$

we obtain

$$
\begin{aligned}
\widetilde{P}_{L y}(k)= & \frac{1}{2 a}\left[b+e^{i k} \widetilde{P}_{L y}(k)+e^{-i k} \widetilde{P}_{L y}(k)\right. \\
& \left.+\widetilde{P}_{L y-1}(k) \widetilde{P}_{L y}(k)\right] .
\end{aligned}
$$

This gives

$$
\widetilde{P}_{L y}(k)=\frac{b}{2 a-2 \cos k-\widetilde{P}_{L y-1}(k)}
$$

which must be solved with the initial condition $\widetilde{P}_{0}(k)=1$ for any $k$. For $L_{y}<\infty$, the continued fraction expansion of Eq. (4) must be solved numerically in general. Even in the zero drift case the expansion converges rapidly, as discussed in the Appendix. In the special case of an infinitely long terrace $L_{y}=\infty, \widetilde{P}_{L y}(k)=\widetilde{P}_{L y-1}(k)$, and Eq. (4) gives

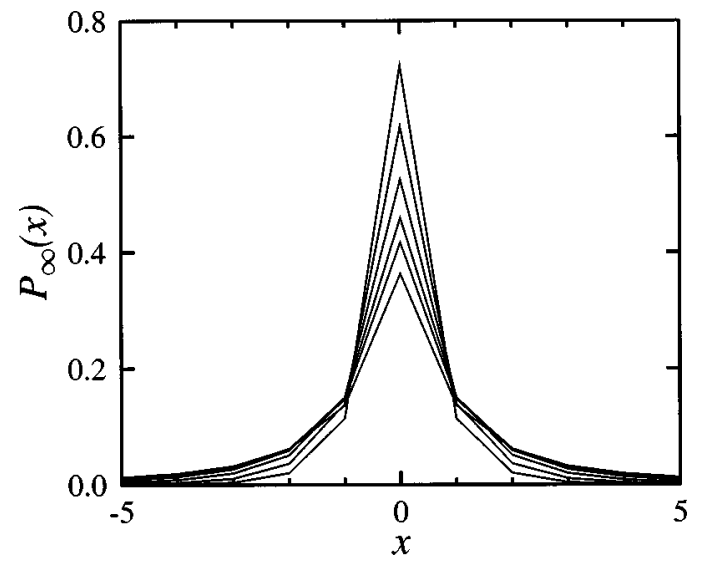

FIG. 3. Probability distribution $P_{\infty}(x)$ for the return position with $L_{x}=10^{4}$ and $L_{y}=\infty$. The drift parameter $l_{d}=1,1 / 2,1 / 4,1 / 8$, $1 / 16$, and 0 (from top to bottom at $x=0$ ). In the limit $l_{d} \rightarrow \infty$ the distribution approaches a $\delta$ function.

$$
\widetilde{P}_{\infty}(k)=a-\cos k-\sqrt{(a-\cos k)^{2}-b} .
$$

In Fig. 3 we show the behavior of $P_{L_{y}}(x)$ for various values of $L_{y}$ and $l_{d}$. In the continuum limit, the tail of this function goes as $x^{-2}$ for the case of zero drift.

In practice, we also need the propagator for a periodic system with a finite width $L_{x}$. This is most easily obtained in the Fourier space by

$$
\begin{aligned}
P_{L x, L y}(x) & =\sum_{r=-\infty}^{\infty} P_{L y}\left(x+r L_{x}\right) \\
& =\sum_{r=-\infty}^{\infty} \frac{1}{2 \pi} \int_{0}^{2 \pi} d k e^{-i k\left(x+r L_{x}\right)} \widetilde{P}_{L y}(k) \\
& =\int_{0}^{2 \pi} d k e^{-i k x} \widetilde{P}_{L y}(k) \frac{1}{2 \pi} \sum_{r=-\infty}^{\infty} e^{-i k r L_{x}} \\
& =\int_{0}^{2 \pi} d k e^{-i k x} \widetilde{P}_{L y}(k) \frac{1}{L_{x}} \sum_{n=-\infty}^{\infty} \delta\left(k-\frac{2 \pi n}{L_{x}}\right) \\
& =\frac{1}{L_{x}} \sum_{n=0}^{L x-1} e^{-i\left(2 \pi n k / L_{x}\right)} \widetilde{P}_{L y}\left(\frac{2 \pi n}{L_{x}}\right) .
\end{aligned}
$$

Numerically, Eq. (6) is easy to implement using the fastFourier-transform algorithm.

\section{Continuum limit of the model}

It is relatively straightforward to write down a continuum description by using the diffusion equation (an electrostatic analogy [23] can also be employed). The probability density of a random walker $u(\vec{r}, t)$ obeys the biased diffusion equation with a source term $\rho(\vec{r}, t)$ :

$$
-\vec{\nabla} \cdot \mathbf{D} \cdot \vec{\nabla} u(\vec{r}, t)+\vec{v} \cdot \vec{\nabla} u(\vec{r}, t)=\rho(\vec{r}, t) .
$$

Using the distribution of the biased random walk, we can derive expressions for the drift term $\vec{v}=\left(0, v_{d}\right)$ and the diagonal elements of the diffusion tensor $\mathbf{D}=D_{\mu \nu}(\mu, \nu=x, y)$ to 
be $v_{d}=-l_{d} /\left(1+l_{d}\right)$, and $D_{x x}=1 /\left(4+4 l_{d}\right)$ and $D_{y y}=1 /(4$ $\left.+4 l_{d}\right)+8 l_{d} /\left(4+4 l_{d}\right)^{2}$. We note that in the model, diffusion is always only slightly anisotropic for $l_{d}>0$, and $D_{x x} / D_{y y}$ $=1 / 3$ for $l_{d} \rightarrow \infty$ [24]. The source term $\rho$ in Eq. (7) is constant over the whole terrace. The boundary conditions are that for the step edge $u=0$ and for the reflecting boundary $\partial u / \partial y=0$. Also, the arrival probability of a random walker at the step edge is proportional to the normal derivative of the probability field $u$. With zero drift $\left(l_{d}=0\right)$, Eq. (7) reduces to the Poisson equation obeyed by many growth models (see, e.g., Refs. $[23,25,26])$. The present sticking rules guarantee that the growing step forms a compact structure, in contrast to the typical diffusion limited aggregation models [23]. It is also evident from the stability analysis of Salditt and Spohn [12] (see also Ref. [11]) that the one-sided diffusion field is highly destabilizing, and the step-edge morphology is always controlled by the instability rather than described by the nonlinear KPZ equation [12].

\section{NUMERICAL RESULTS}

\section{A. Ledge roughness}

We have performed extensive Monte Carlo simulations of the model with the algorithm described in Sec. II. In this work, we consider the case of finite $l_{d}$ only [27]. First, we discuss results for the roughness of the growing ledge on an infinitely long terrace $\left(L_{y}=\infty\right)$ with a large value of $L_{x}$ $=10^{4}$. In this case, after a short initial transient the undulations of the ledge grow and fingerlike structures emerge, separated by deep cracks. The cracks deepen and the fingers themselves coarsen at the expense of other fingers. In Fig. 4, we show a sequence of typical successive configurations for different values of $l_{d}$. We find that the width $w(t)$ of the interface associated with the ledge follows power law behavior

$$
w(t) \equiv\left\langle\overline{[h(x, t)-\bar{h}(t)]^{2}}\right\rangle^{1 / 2}=A t^{\beta_{1}},
$$

where the brackets and the overbar denote an average over the configurations and over each finite system, respectively. The height variable $h(x, t)$ is the column height of the ledge as measured from $y=0$. Numerically, we find that the width $w(t)$ grows linearly with $\beta_{1}=1.0 \pm 0.01$ and its slope $A\left(l_{d}\right)$ depends on the drift $l_{d}$ (Fig. 5). Linear growth can be understood qualitatively, since particles arriving at the vertical section of the ledge do not contribute significantly to the ledge roughness. The increase in the roughness is mainly due to particles that stick on top of the columns, and thus the width grows proportional to the total particle number, i.e., time. The change in the growth rate is also easy to explain qualitatively. With small drifts, only the top of the finger grows and very few particles reach the bottom of the cracks. With larger drifts, the probability of reaching the bottom increases, and thus $w$ increases more slowly (see also Fig. 4).

The value of $\beta_{1}=1$ is consistent with the theory of Elkinani and Villain [28] for a simple 1D Zeno model of MBE growth with step-edge barriers. Instead of ledges, they consider deposition of adatoms on a stepped surface with diffusion. They show that with strong step-edge barriers, deep cracks are formed on the surface whose depth grows linearly
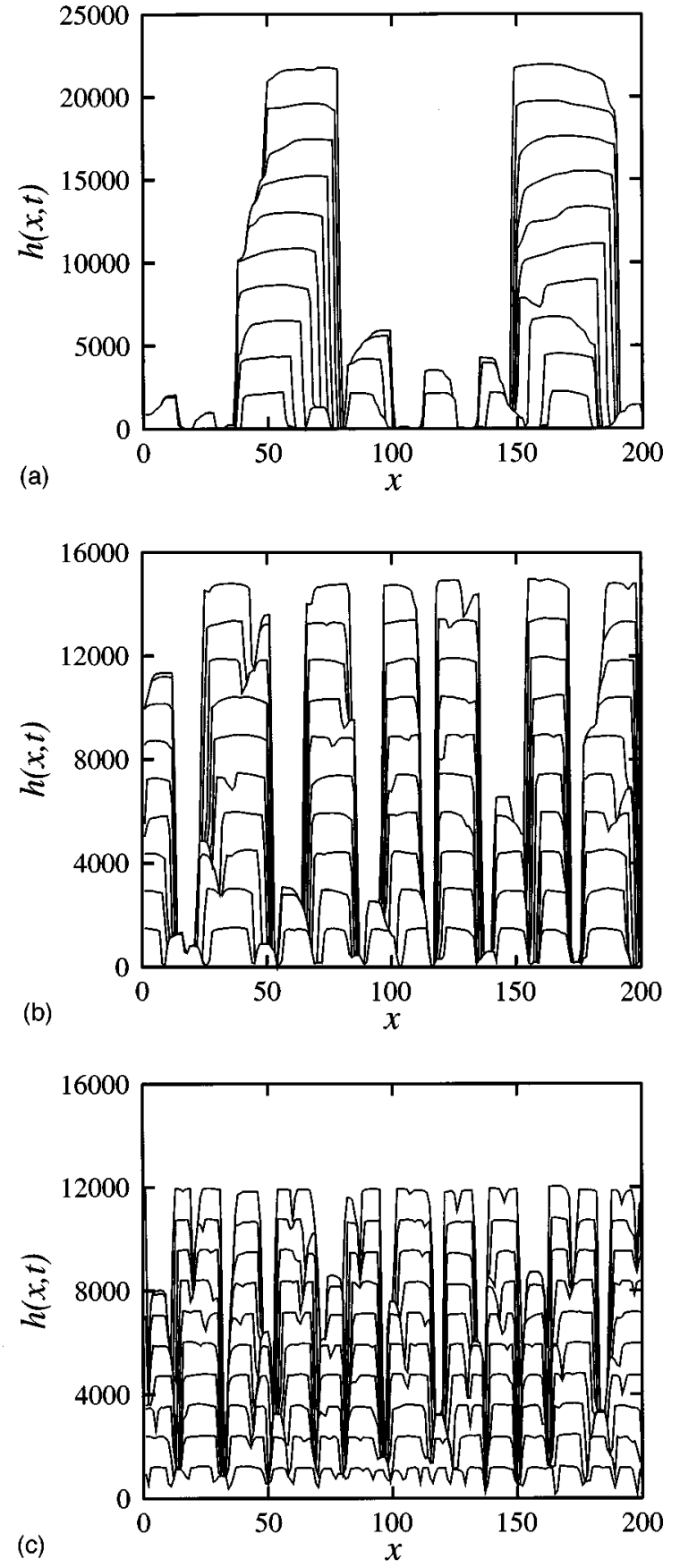

FIG. 4. Ten consecutive step profiles from the growth model with $L_{x}=10^{4}$ and $L_{y}=\infty$ at $t=1000,2000, \ldots, 10^{4}$ with the drift (a) $l_{d}=1 / 4$, (b) $l_{d}=1$, and (c) $l_{d}=4$. Only part of the system is shown.

in time. In this case, the deposition noise is not relevant and this result can be obtained from a deterministic model.

To study the effect of a finite terrace length $L_{y}<\infty$, we have simulated the model with $l_{d}=1 / 4, L_{x}=10^{4}$, and $L_{y}$ $=50,70,100,140,200$, and 500. Due to the fact that in such finite systems the relative proportion of the flux deposited in between the fingers increases with time, the width $w(t)$ eventually saturates to an $L_{y}$-dependent value, but does not saturate as a function of $L_{x}$. We find that the width satisfies the scaling ansatz of Family-Vicsek [2,29]:

$$
w\left(L_{y}, t\right)=t^{\chi_{1} / z_{1}} f_{1}\left(L_{y} / t^{1 / z_{1}}\right),
$$




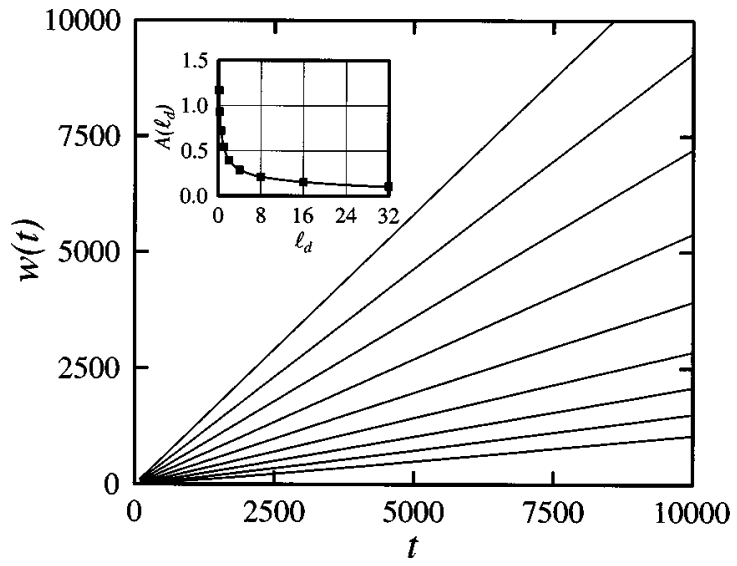

FIG. 5. The step width $w(t)$ for $l_{d}=1 / 8,1 / 4,1 / 2,1,2,4,8,16$, and 32 (from top to bottom) with $L_{x}=10^{4}$ and $L_{y}=\infty$. The slope $A$ as a function of the drift $l_{d}$ is shown in the inset.

where the scaling function $f_{1}(x)$ behaves as

$$
f_{1}(x) \sim \begin{cases}\text { const, } & x \gg 1, \\ x^{\chi_{1},}, & x \ll 1 .\end{cases}
$$

The exponent $\chi_{1}$ characterizes the surface morphology in the saturated regime $w\left(L_{y}\right) \sim L_{y}^{\chi_{1}}$, and the crossover time $t_{\text {sat }} \sim L_{y}^{z_{1}}$ determines where the saturation takes over. The growth exponent for $t \ll t_{\text {sat }}$ is $\beta_{1}=\chi_{1} / z_{1}$. We find that setting $\beta_{1}=1, z_{1}=1.00 \pm 0.03$ collapses our data best to a single scaling function shown in Fig. 6. We have also obtained the exponent $\chi_{1}$ by fitting to the saturated width $w\left(L_{y}\right)$ and find that $\chi_{1}=0.96 \pm 0.02$.

\section{B. Finger coarsening}

In our model, diffusion along the ledge is limited by infinitely strong barriers, since the particles can never go around corners. This is basically the same effect as step barriers

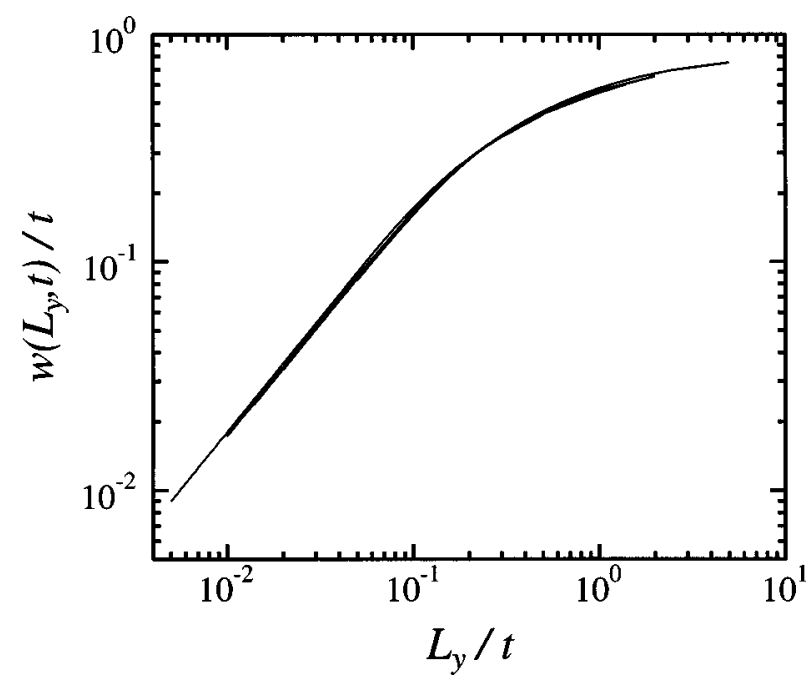

FIG. 6. Scaling function $f_{1}$ of Eq. (10) for the step width $w\left(L_{y}, t\right)$ with $L_{y}=50,70,100,140,200$, and 500. Good scaling is obtained with $\beta_{1}=1$ and $z_{1}=1$. The drift $l_{d}=1 / 4$ and the lateral lattice size $L_{x}=10^{4}$.

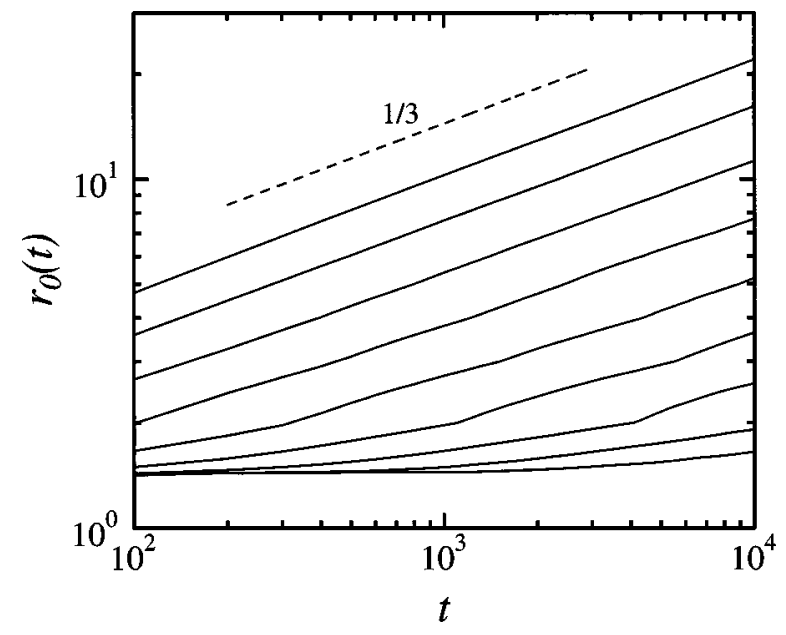

FIG. 7. The finger width $r_{0}(t)$ for $l_{d}=1 / 8,1 / 4,1 / 2,1,2,4,8$, 16 , and 32 (from top to bottom) with $L_{x}=10^{4}$ and $L_{y}=\infty$. The dashed line indicates a slope of $1 / 3$.

along the surface of the 1D Zeno model. However, since in our model there is a real diffusion field surrounding the fingers on the terrace, additional finger coarsening [30] takes place as is evident in the configurations of Fig. 4. For a finite system with $L_{x}<\infty$, this eventually leads to a configuration where there is only one finger present. To investigate the temporal scaling of the thickness of the fingers, we have studied how the first zero of the Green's function at $r$ $=r_{0}(t)$,

$$
G(r, t)=\left\langle\frac{1}{N} \sum_{x} h(x+r, t) h(x, t)-\bar{h}(t)^{2}\right\rangle,
$$

behaves as a function of time. The behavior of $r_{0}(t)$ should indicate the existence of a characteristic, time-dependent correlation length in the direction perpendicular to the direction of growth. In Fig. 7 we show $r_{0}(t)$ for several values of $l_{d}$ when $L_{x}=10^{4}$ and $L_{y}=\infty$. To a good degree of accuracy, we find that $r_{0}(t) \sim t^{\beta_{r}}$, with the value $\beta_{r}=0.32 \pm 0.01$ for drifts varying from $1 / 8$ to 32 . There is, however, a long crossover regime at the beginning of the growth that depends on the drift, being longer for larger drift values.

It is also interesting to study the scaling of the Green's function. Asymptotically, we expect $G(r, t)$ to scale as [31]

$$
G(r, t)=t^{-2 \beta_{1}} g_{l_{d}}\left(t^{-\beta_{r} r}\right),
$$

where $g_{l_{d}}(x)$ is a new scaling function associated with the coarsening process. In Fig. 8 we show scaling of the data for $G(r, t)$, with a very good data collapse obtained with $\beta_{1}=1$ and $\beta_{r}=1 / 3$ [32]. It is interesting to note that the finger coarsening in the present model follows the same power law of $1 / 3$ as model $B$, which describes domain coarsening due to long-range diffusion [31,33]. However, although qualitatively similar, the present scaling function depicted in Fig. 8 is quantitatively different from that of model B [33]. The exponent $1 / 3$ also appears in models of noisedriven coarsening of mounds in 1D surface growth where slope selection occurs because of step-edge barriers $[7,26]$.

The finite-size scaling of $r_{0}$ is different from that of the width $w$, since for a system with a finite terrace width $L_{x}$ 


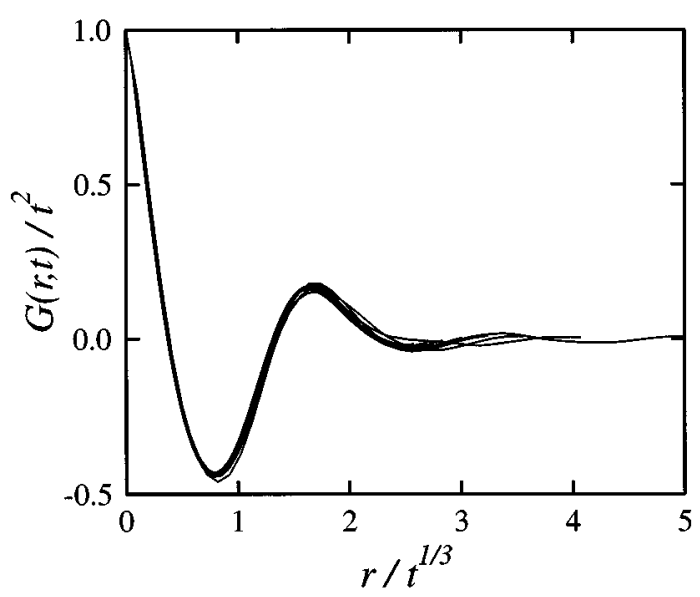

FIG. 8. Scaling function $g_{l_{d}}$ of Eq. (13) for $G(r, t)$ with $L_{x}$ $=10^{4}$ and $l_{d}=1$ at ten different times $t=1000,2000, \ldots, 10^{4}$, with $\beta_{1}=1$ and $\beta_{r}=1 / 3$.

$<\infty$ but with $L_{y}=\infty$, the late-time configuration consists of one finger only, whose vertical roughness $w$ keeps on growing linearly but whose $r_{0}$ saturates. This introduces a new exponent $z_{2}$ that controls the saturation of $r_{0}$ in the $x$ direction. On the other hand, for $L_{x}=\infty$ and $L_{y}<\infty$, both $w$ and $r_{0}$ saturate, and their saturation must be characterized by the same exponent $z_{1}$ in Eq. (9). Thus, for the general case of both $L_{x}, L_{y}<\infty$, we expect the following scaling form to hold:

$$
r_{0}\left(L_{x}, L_{y}, t\right)=t^{\beta_{r}} f_{r}\left(\frac{L_{x}}{t^{1 / z_{2}}}, \frac{L_{y}}{t^{1 / z_{1}}}\right)
$$

We will not study the whole scaling function $f_{r}(x, y)$ here but consider the effects of a finite $L_{x}$ and $L_{y}$ separately [34]. For $L_{y}=\infty$, we can again write down the Family-Viscek form as

$$
r_{0}\left(L_{x}, t\right)=t^{\chi_{2} / z_{2}} f_{2}\left(L_{x} / t^{1 / z_{2}}\right)
$$

where now $\beta_{r} \equiv \chi_{2} / z_{2}$, and the scaling function $f_{2}$ has the same limits as $f_{1}$, but now with a new roughness exponent $\chi_{2}$. We have simulated the model with $L_{x}=20,50,100$, and 200 using the drift $l_{d}=1$. Because of the single finger final configuration, there are large fluctuations in the data and thus we have determined the saturation exponent $\chi_{2}$ by estimating the saturated width $r_{0}\left(L_{x}\right)$ directly for various values of $L_{x}$. From the data, our best estimate is $\chi_{2}=1.02 \pm 0.01$, i.e., the width of the final finger grows as the horizontal system size. Together with $\beta_{r}=0.33$ this implies that $z_{2}=3.0$.

In the case of a finite $L_{y}$, we expect that the scaling form satisfies

$$
r_{0}\left(L_{y}, t\right)=t^{\chi_{3} / z_{1}} f_{3}\left(L_{y} / t^{1 / z_{1}}\right)
$$

where $\beta_{r}$ must now satisfy the relation $\beta_{r}=\chi_{3} / z_{1}$, with $\chi_{3}$ being another new roughness exponent. The limits of $f_{3}$ and $f_{1}$ are again of the same form. By using system sizes $L_{y}=$ $50,70,100,140,200$, and 500 with the drift $l_{d}=1 / 4$, our data collapse to the scaling form shown in Fig. 9 with $\chi_{3}$ $=0.33 \pm 0.01$ and $z_{1}=1.00 \pm 0.03$. Moreover, we have ob-

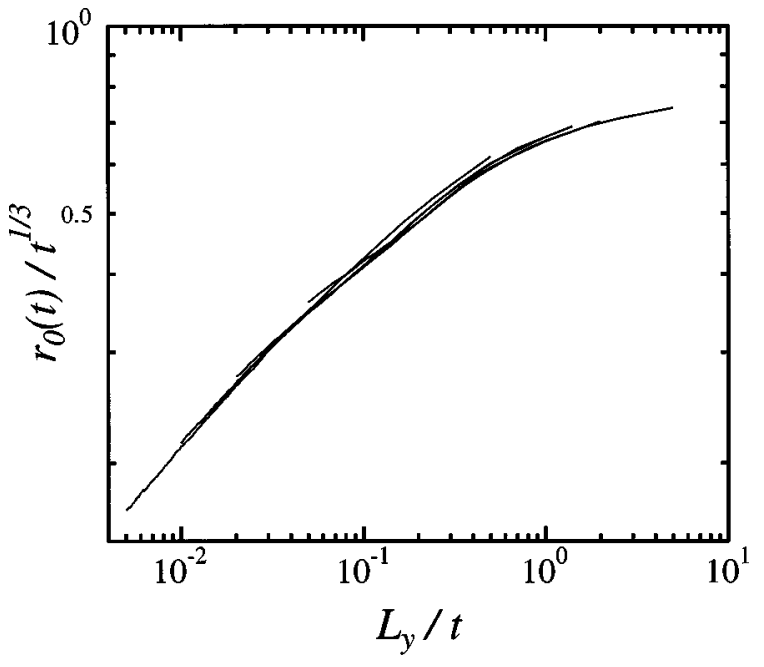

FIG. 9. Scaling function $f_{3}$ of Eq. (16) for the finger width $r_{0}$ with the terrace length $L_{y}=50,70,100,140,200$, and 500. The drift $l_{d}=1 / 4$ and the lateral lattice size $L_{x}=10^{4}$, and $\beta_{r}=1 / 3$ and $z_{1}=1$.

tained another estimate of the new saturation exponent $\chi_{3}$ by estimating the saturated width $r_{0}\left(L_{y}\right)$ and indeed verify that $\chi_{3}=0.34 \pm 0.02$.

\section{SUMMARY AND CONCLUSIONS}

In summary, in this work we have introduced and examined a very simple model for the growth of an isolated step with infinitely strong step-edge barriers. The destabilizing effect of the one-sided biased diffusion field coupled with strongly anisotropic adatom dynamics makes the ledge morphologically unstable, with fingerlike structures developing separated by deep cracks. After an initial early-time transient the fingers coarsen as $t^{0.33}$ and the width of the ledge grows linearly. For an infinitely wide and long terrace, the fingers eventually become needlelike. We have also studied the finite-size scaling of both the coarsening and the width of the ledge in detail, and determined the corresponding scaling exponents.

Recently, Pierre-Louis et al. [14] have considered in detail a more realistic model of step train growth in the case of weak desorption, and one-side attachment. As in the present case, they find that the step morphology is linearly unstable, but now the individual step widths grow $\propto t^{1 / 2}$, with the steps "locked in", together. In this regime, there is no step coarsening, either. Thus, we expect our model to be relevant only for the case where the steps are well isolated, and detachment from step edges can be neglected.

Experimentally, growth of steps on slightly miscut $\mathrm{Si}(001)$ surfaces has been studied, with the claimed result that the step roughening is consistent with the KPZ prediction [35]. However, at least superficially the steps depicted in Ref. [35] appear to develop fingerlike structures separated by deep grooves characteristic of the unstable regime studied here and in Ref. [14]. It would be interesting to carry out more systematic studies of roughening of widely spaced steps on semicondutor surfaces to characterize the nature of the instability. 


$$
\widetilde{P}_{L y}(\omega)=\sum_{t=-\infty}^{\infty} e^{-i \omega t} P_{L y}(t)
$$

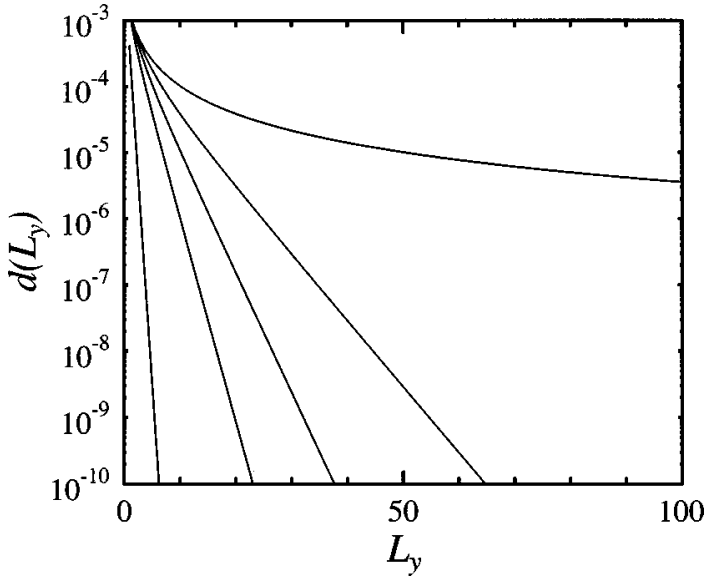

FIG. 10. Root mean square deviation $d$ of the propagator $P_{L y}$ from $P_{\infty}$ with $L_{x}=10^{4}$ for $l_{d}=0,1 / 16,1 / 8,1 / 4$, and 4 (from top to bottom), shown as a function of the distance $L_{y}$ to the reflecting boundary.

\section{ACKNOWLEDGMENTS}

T.A-N. wishes to thank J. Krug and M. Rost for useful discussions. This work was supported in part by the Academy of Finland, and by NSF Grant No. DMR-9222812.

\section{APPENDIX}

In this appendix, we calculate explicitly the average arrival time $t_{\text {arr }}$ of a walker to demonstrate the need to use the present algorithm. We will also discuss the convergence of the probability distribution for finite terraces with $L_{y}<\infty$. To begin with, the distribution for the number of steps or the distribution of the first passage time $P_{L y}(t)$, can be calculated similar to the spatial distribution of Eq. (1) by the recursion

$$
\begin{aligned}
P_{L y}(t)= & \frac{1}{2 a} \sum_{s=1}^{\infty} \delta_{t-s, 1}\left(b \delta_{s, 0}+2 P_{L y}(s)\right. \\
& \left.+\sum_{u=1}^{\infty} P_{L y}(s-u) P_{L y-1}(u)\right) .
\end{aligned}
$$

Using the temporal Fourier transform we obtain the characteristic function as

$$
\widetilde{P}_{L y}(\omega)=\frac{b}{2 a e^{i \omega}-2-\widetilde{P}_{L y-1}(\omega)},
$$

which can be solved with the initial value $\widetilde{P}_{0}(\omega)=1$. Again, $\widetilde{P}_{L y}(\omega)=\widetilde{P}_{L y-1}(\omega)$ when $L_{y} \rightarrow \infty$ and $\widetilde{P}_{\infty}(\omega)$ can be obtained. The average arrival time is proportional to the first derivative of the characteristic function at $\omega=0$ by

$$
\begin{aligned}
t_{\mathrm{arr}} & =\sum_{t=1}^{\infty} t P(t)=\left.i \frac{d \widetilde{P}(\omega)}{d \omega}\right|_{\omega=0} \\
& =-\frac{\widetilde{P}_{L y}^{2}(0)}{b}\left(2 i a-\left.\frac{d \widetilde{P}_{L y-1}(\omega)}{d \omega}\right|_{\omega=0}\right) \\
& =\left.\frac{d \widetilde{P}_{L y}(\omega)}{d \omega}\right|_{\omega=0}=-2 i a \sum_{n=1}^{L y}\left(\frac{1}{b}\right)^{n} \\
& =\left(1+\frac{1}{l_{d}}\right)\left(1-\frac{1}{\left(1+4 l_{d}\right)^{L y}}\right)
\end{aligned}
$$

when $l_{d}>0$. In the infinite terrace limit $\left(L_{y} \rightarrow \infty\right), t_{\text {arr }}=1$ $+1 / l_{d}$, while for $l_{d}=0$ it is easy to show that $t_{\mathrm{arr}}=4 L_{y}$. Thus, the return time quickly becomes prohibitively large for large systems and small values of the drift, making bruteforce Monte Carlo simulations difficult. On the other hand, for drifts larger than unity, no significant reduction in computer time can be obtained with the new algorithm.

Finally, to estimate the convergence of the probability distribution $P_{L y}(x)$ towards its asymptotic limit as a function of the terrace length $L_{y}$ for any $L_{x}$, we can define the deviation $d$ by

$$
d^{2}\left(L_{x}, L_{y}, l_{d}\right)=\frac{1}{L_{x}} \sum_{n=0}^{L_{x}-1}\left[\widetilde{P}_{L y}\left(\frac{n}{2 \pi L_{x}}\right)-\widetilde{P}_{\infty}\left(\frac{n}{2 \pi L_{x}}\right)\right]^{2},
$$

assuming a periodic system in the $x$ direction. In Fig. 10, we show the deviation for various values of the drift as a function of $L_{y}$.
[1] D. J. Eaglesham, J. Appl. Phys. 77, 3597 (1995).

[2] A.-L. Barabási and H. E. Stanley, Fractal Concepts in Surface Growth (Cambridge University Press, City, 1995).

[3] Reflection High Energy Electron Diffraction and Reflection Electron Imaging of Surfaces, edited by P. K. Larsen and P. J. Dobson (Plenum Press, New York, 1988).

[4] W. K. Burton, N. Cabrera, and F. C. Frank, Philos. Trans. R. Soc. London, Ser. A 243, 299 (1951).

[5] R. Ghez and S. S. Iyer, IBM J. Res. Dev. 32, 804 (1988).

[6] R. L. Schwoebel, J. Appl. Phys. 44, 614 (1969).

[7] J. Krug, M. Plischke, and M. Siegert, Phys. Rev. Lett. 70, 3271 (1993); J. Krug and H. Dobbs, ibid. 73, 1947 (1994); M. Sieg- ert and M. Plischke, Phys. Rev. E 50, 917 (1994).

[8] M. Siegert and M. Plischke, Phys. Rev. Lett. 73, 1517 (1994).

[9] M. Kotrla and P. Smilauer, Acta. Phys. Slov. 44, 237 (1994);

F. Liu and H. Metiu, Phys. Rev. E 49, 2601 (1994); C. J. Lanczycki and S. Das Sarma, Phys. Rev. E 50, 213 (1994); S. A. Chalmers, J. Y. Tsao, and A. C. Gossard, J. Appl. Phys. 73, 7351 (1993); D. E. Wolf, Phys. Rev. Lett. 67, 1783 (1991); L.-H. Tang and T. Nattermann, ibid. 66, 2899 (1991); A. K. Myers-Beaghton and D. D. Vvedensky, Phys. Rev. B 42, 9720 (1990).

[10] I. Bena, C. Misbah, and A. Valence, Phys. Rev. B 47, 7408 
(1993); A. Karma and C. Misbah, Phys. Rev. Lett. 71, 3810 (1993).

[11] G. S. Bales and A. Zangwill, Phys. Rev. B 41, 5500 (1990).

[12] T. Salditt and H. Spohn, Phys. Rev. E 47, 3524 (1993).

[13] O. Pierre-Louis and C. Misbah, Phys. Rev. Lett. 76, 4761 (1996).

[14] O. Pierre-Louis, C. Misbah, Y. Saito, J. Krug, and P. Politi (unpublished).

[15] M. Kardar, G. Parisi, and Y.-C. Zhang, Phys. Rev. Lett. 56, 889 (1986).

[16] H. J. W. Zandvliet and H. B. Elswijk, Phys. Rev. B 48, 14269 (1993).

[17] In the case of one-particle diffusion, the drift term is not present strictly speaking since the step does not move. In our model $l_{d}$ may be considered as a finite-density correction to this limit.

[18] C. Roland and G. H. Gilmer, Phys. Rev. Lett. 67, 3188 (1991); Phys. Rev. B 46, 13428 (1992); D. Srivastava and B. Garrison, ibid. 47, 4464 (1993); C. P. Toh, S. H. Seow, and C. K. Ong, Surf. Sci. 292, 114 (1993).

[19] Q.-M. Zhang, C. Roland, P. Boguslawski, and J. Bernhole, Phys. Rev. Lett. 75, 101 (1995).

[20] J. Hoeven, J. M. Lenssinck, D. Dijkkamp, E. J. van Loenen, and J. Dieleman, Phys. Rev. Lett. 63, 1830 (1989); Y.-W. Mo, B. S. Swartzentruber, R. Kariotis, M. B. Webb, and M. G. Lagally, ibid. 63, 2393 (1989); Y. W. Mo, J. Kleiner, M. B. Webb, and M. G. Lagally, ibid. 66, 1998 (1991); Y.-W. Mo and M. G. Lagally, Surf. Sci. 248, 313 (1991); Y.-W. Mo, J. Kleiner, M. B. Webb, and M. G. Lagally, ibid. 268, 275 (1992); B. Voigtländer, T. Weber, P. Ŝmilauer, and D. E. Wolf, Phys. Rev. Lett. 78, 2164 (1997).

[21] J. E. Griffith, G. P. Kochanski, J. A. Gubby, and P. E. Wierenga, J. Vac. Sci. Technol. 7, 1914 (1989).

[22] G. Brocks, P. J. Kelly, and R. Car, Phys. Rev. Lett. 66, 1729 (1991); C. P. Toh and C. K. Ong, Phys. Rev. B 45, 11120 (1992).

[23] T. A. Witten and L. M. Sander, Phys. Rev. Lett. 47, 1400 (1981).

[24] On a $\mathrm{Si}(001)$ terrace, diffusion along the dimer rows is much faster than across them. For $l_{d}>0$, however, the step morphology is controlled by the drift term, and thus the exact value of the ratio $D_{x x} / D_{y y}$ is not important.
[25] J. Krug, K. Kassner, P. Meakin, and F. Family, Europhys. Lett. 24, 527 (1993).

[26] J. Krug, Adv. Phys. 46, 139 (1997).

[27] The zero drift limit $l_{d} \rightarrow 0$ is rather subtle in the present model. For systems with finite $L_{x}$, we find that asymptotically $w(t)$ $\sim t$. However, the behavior of the finger coarsening is somewhat different from the case of $l_{d}>0$. We do find an intermediate time regime where $r_{0}(t) \sim t^{0.33}$, but for larger systems there seems to be a late-time crossover to a larger effective coarsening exponent. In this regime, our model becomes equivalent to the "Laplacian needle model A'" of Krug et al. [25]. In the needle model, the average distance between the needles grows like $h_{a} / \ln \left(h_{a}\right)$, where $h_{a}$ is the height of "active"' needles [26]. This would mean that $r_{0}(t) \sim t / \ln (t)$, which is qualitatively supported by our simulation results for $l_{d}=0$.

[28] I. Elkinani and J. Villain, J. Phys. I 4, 949 (1994).

[29] F. Family and T. Vicsek, J. Phys. A 18, L75 (1985).

[30] P. Politi and J. Villain, Phys. Rev. B 54, 5114 (1996).

[31] A. J. Bray, Adv. Phys. 43, 357 (1994).

[32] As defined in Eq. (13), the scaling function $g_{l_{d}}(x)$ depends on the drift $l_{d}$. We can also write the scaling form as $G(r, t)$ $=w^{2}\left(l_{d}, t\right) g\left(r / r_{0}\left(l_{d}, t\right)\right)$, in which case the scaling plots for the new function $g(x)$ collapse within the accuracy of our data for the values of the drift studied, namely $l_{d}=1 / 8,1 / 4,1 / 2$, and 1.

[33] T. M. Rogers, K. R. Elder, and R. C. Desai, Phys. Rev. B 37, 9638 (1988).

[34] In the long-time limit, for an infinite system $\left(L_{x}=L_{y}=\infty\right)$ the aspect ratio $r_{0} / w$ approaches zero, and the fingers become needlelike even for finite values of $l_{d}$. In the needle model, the penetration probability of the diffusing particles between needles decays exponentially as $\exp (-\pi r / \xi)$ [25], where the distance $r$ is measured from the top of the needles and $\xi$ is the distance between two needles. In our model with a finite drift parameter $l_{d}$, the corresponding result can be shown to be $\exp \left\{-\left(1 / 2 D_{y y}\right)\left[\sqrt{4 D_{x x} D_{y y} \pi^{2} / \xi^{2}+v_{d}^{2}\left(l_{d}\right)}+v_{d}\left(l_{d}\right)\right] r\right\}$, where $v_{d}(l), D_{x x}$, and $D_{y y}$ are defined in the text. For zero drift (where $v_{d}=0$, and $D_{y y}=D_{x x}$ ), this correctly reduces to the previous result.

[35] F. Wu, S. G. Jaloviar, D. E. Savage, and M. G. Lagally, Phys. Rev. Lett. 71, 4190 (1993). 